\title{
Numerical investigations of stress shielding in total hip prostheses
}

\author{
B-A Behrens ${ }^{1}$, C J Wirth ${ }^{2}$, H Windhagen ${ }^{2}$, I Nolte ${ }^{3}$, A Meyer-Lindenberg ${ }^{3}$, and A Bouguecha ${ }^{1 *}$ \\ ${ }^{1}$ Institute of Metal Forming and Metal-Forming Machines, Leibniz Universität Hannover, Hannover, Germany \\ ${ }^{2}$ Department of Orthopaedics, Medical University of Hannover, Hannover, Germany \\ ${ }^{3}$ Small Animal Clinic, University of Veterinary Medicine Foundation, Hannover, Germany
}

The manuscript was received on 20 December 2005 and was accepted after revision for publication on 11 January 2008.

DOI: 10.1243/09544119JEIM139

\begin{abstract}
Aseptic loosening of the prosthesis is still a problem in artificial joint implants. The loosening can be caused by, among other factors, resorption of the bone surrounding the prosthesis owing to stress shielding. In order to find out the influence of the prosthesis type on post-operative stress shielding, a static finite element analysis of a femur provided with the conventional uncemented stem BICONTACT and of one with the femoral neck prosthesis SPIRON was carried out. Strain energy densities and maximal principal strain distributions were calculated and compared with the physiological situation. Here, stress shielding was demonstrated in both periprosthetic femora. To determine the areas of the stress shielding, the bone in each FE model was subdivided into three regions of interest (ROI): proximal, diaphyseal, and distal. The numerical computations show stress shielding in the proximal ROI of both periprosthetic femora. Diaphyseally, the femoral neck prosthesis SPIRON, in contrast to the conventional uncemented long-stem prosthesis BICONTACT, causes no decrease in the strain distribution and thus no stress shielding. Distally, no change in the load distribution of either periprosthetic femur could be found, compared with the physiological situation.
\end{abstract}

Keywords: human femur, stress shielding, finite element analysis, total hip arthroplasty, strain energy density, maximal principal strain

\section{BACKGROUND}

Total hip arthroplasty (THA) is a very successful routine treatment for severe degenerative or traumatic changes to the hip joint $[\mathbf{1}]$. As cortical and particularly cancellous bone, from a mechanical point of view, is essentially less stiff than the metal material of the prosthesis, a partial unloading of the bone tissue occurs surrounding an implanted conventional femoral prosthesis component. This phenomenon is called stress shielding [2]. Herein, the bone adapts to the changed stress distribution by remodelling, as hypothesized by Wolff's law [3], resulting in bone resorption which can contribute to a loosening of the implant [4]. Bone resorption in

*Corresponding author: Numerical Methods, Institute of Metal Forming and Metal-Forming Machines, Leibniz Universität Hannover, Garbsen, Hannover 30823, Germany. email: bouguecha@ ifum.uni-hannover.de stress-shielded regions of the periprosthetic femur has been shown by several studies in dogs [5] and humans $[4,6,7]$. For the patient, the consequence of prosthesis loosening may be severe pain which usually results in cost-intensive revision surgery [8]. Therefore, maintaining a load distribution in the femur as near as possible to the physiological situation is an important factor for permanent anchorage of the hip prosthesis in the bone. It is hypothesized that, with an appropriate prosthesis shape, stress shielding and the resulting bone resorption could be reduced and long-term survival rates of femoral components could be further improved.

Nowadays, finite element analysis (FEA) has been established as a time-saving and cost-efficient computing method in biomedical engineering. On the one hand it can be used to design and optimize prostheses [9] and on the other hand it can be used to examine the change in the physiological load distribution after THA [10-13]. 
In this work, this method is used to determine the influence of the prosthesis type on post-operative stress shielding. Hence, a conventional uncemented stem and a femoral neck prosthesis are compared regarding the resulting change in the load distribution in the periprosthetic bone.

\section{METHODS}

For the numerical calculations, a three-dimensional FE design of a femur was developed on the basis of computed tomography (CT) (SOMATOM PLUS 4, Siemens, Munich, Germany) data of a human patient. The examination was performed in preparation for robot-assisted total hip replacement. After informed consent was obtained, the caudal pelvis and the femur were scanned with a slice thickness of $2 \mathrm{~mm}$. With the image analysis program Analyze (Mayo Clinic Biomedical Imaging Resource, Jacksonville, Florida, USA), three-dimensional surfaces of the image data were created in stereolithography format. By using the preprocessor software MSC.Patran (MSC.Software, Santa Ana, California, USA), a solid finite element model was generated. The model was meshed using ten-noded tetrahedral elements.

In order to describe the elastic behaviour of the cortical bone, an orthotropic homogeneous material law was implemented in the FE analysis system MSC.Marc (MSC.Software, Santa Ana, California, USA). These material properties of femoral bone were taken from the literature [14]. For the cancellous bone, however, a homogeneous isotropic material law was chosen, and average values from the experiments by Bini et al. [15] were used.

First, the physiological load situation was determined by a static simulation of an intact femur. The results serve as reference data to evaluate the change in the load distribution in the bone after THA. As femoral strains are different when muscle forces are neglected [16], a simplified loading regime was used, modelling the forces of the abductors, the tensor fasciae latae, and the vastus lateralis. The muscle forces and hip resultant force $\left(F_{1}, F_{2}, F_{3}, F_{4}, F_{5}\right)$ representing a human weighing $85 \mathrm{~kg}$ were taken from the work of Heller and coworkers [17]. These muscle forces conform to those that are effective on the femur of a human at a maximum load while walking. The distal end of the femur was fixed using one thrust and four floating bearings (Fig. 1).

For the simulation of the prosthesis-containing femur, two different types of prosthesis were chosen. As a conventional prosthesis with a long shaft, the femoral component of BICONTACT (Aesculap AG and Co. KG, Tuttlingen, Germany) was used. As an example of a femoral neck implant, the SPIRON prosthesis (Arge Medizintechnik GmbH, Hannover, Germany) was modelled.

For the numerical simulations of the interconnection between bone and prosthesis, a threedimensional FE model was constructed from computer aided design (CAD) data of both prostheses, which were provided by the respective producers. These prostheses were also discretized with ten-noded tetrahedra. The implantation of the prostheses into the femur was accomplished with the preprocessor HyperMesh (Altair Engineering $\mathrm{GmbH}$, Böblingen, Germany) according to the surgery instructions of the respective manufacturer and with X-rays from the patient data of the Department of Orthopaedics, Medical University of Hannover (Fig. 2). The BICONTACT stem is coated with pure titanium in the proximal area; as the intention and to simulate the situation directly after surgery, when no bony ingrowth of the implant has occurred, the prosthesis was bonded to the bone only in this coated region.

For the FE simulation of the interconnection between bone and prosthesis, the same boundary conditions (joint and muscle forces) were chosen as for the simulation of the intact femur. To eliminate the influence of different implant materials on the results, both prostheses were modelled using a homogeneous and isotropic material law for titanium $\left(E=110000 \mathrm{~N} / \mathrm{mm}^{2}\right)$.

The absolute elastic three-dimensional calculations of the strain energy density (SED) and the maximal principal strain (MPS) were performed with the solver MSC.Marc.

\section{RESULTS}

Figure 3 shows the SED distribution in the intact bone (left), acting as a reference, the femur provided with the convential uncemented stem BICONTACT (middle), and the one with the neck prosthesis SPIRON (right). The maximum SED in the intact bone is $0.06 \mathrm{MJ} / \mathrm{m}^{3}$.

A change in the SED distribution in both proximal periprosthetic femora, compared with the physiological situation, can be noticed. A decline in the SED values implies a decrease in the load and thus stress shielding.

For better comparison of the results, the bone in each FE model was subdivided into three regions of interest (ROI): proximal, diaphyseal, and distal (Fig. 3). 


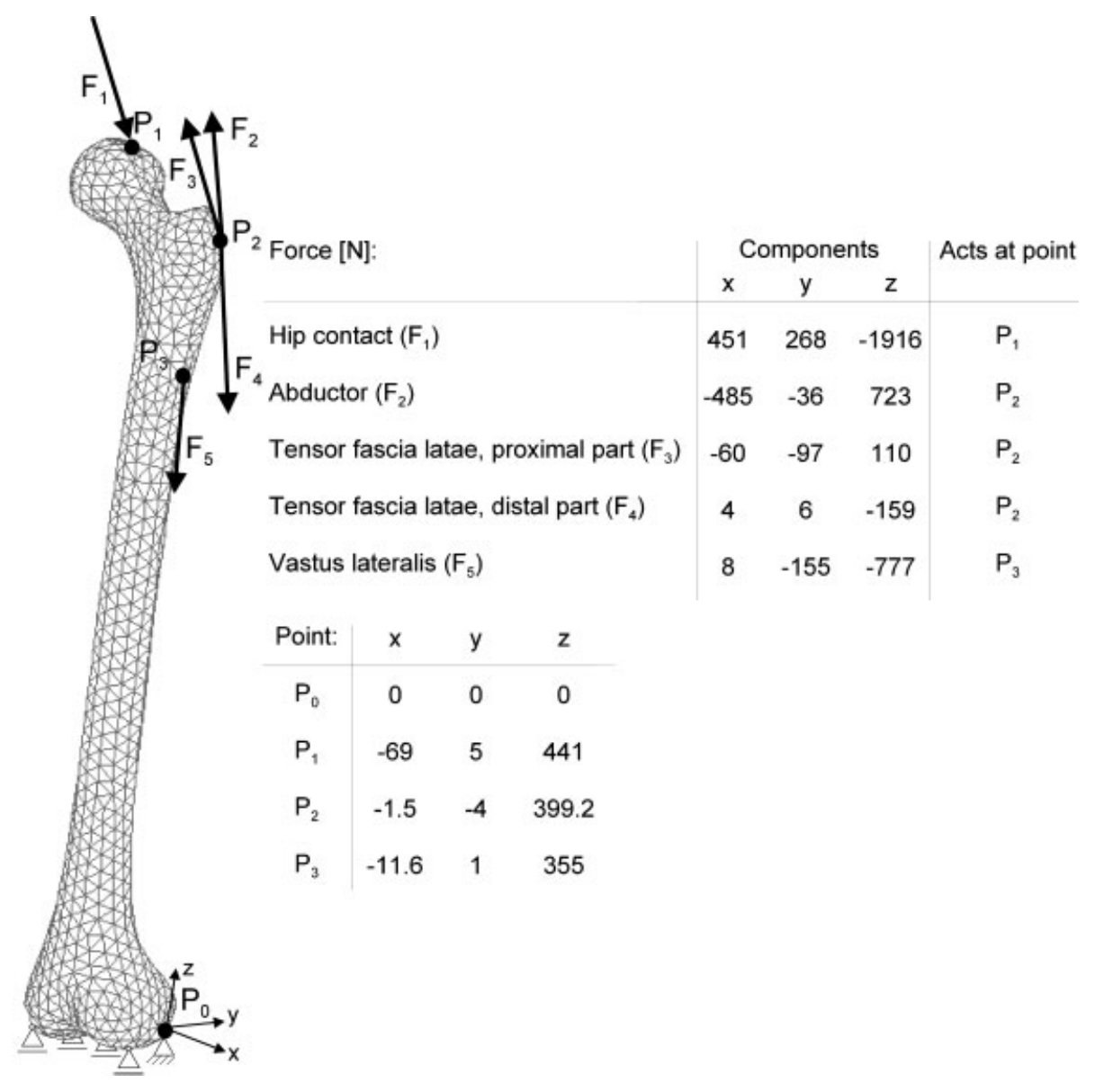

Fig. 1 Loading of the femur (data obtained from reference [17])

Herein, the proximal ROI displays the upper part of the femur and also covers BICONTACT's coated area and the femoral neck prosthesis SPIRON, while the diaphyseal ROI ends distal to BICONTACT's stem. The distal ROI describes the remaining area of the femur.

Within this study, the MPS was calculated as well. In order to illustrate the MPS distributions within the femur, a transverse section (TS) across the bone is shown as an example for each ROI (Fig. 4). The figure clearly shows that a THA in the intertrochanteric TS causes a change in the distribution of the MPS in the bone surrounding the prosthesis. Moreover, there is no noticeable change in the diaphyseal TS and none in the distal one either. It was also demonstrated in all three models that the maximum MPS value does not exceed 0.002 .

In order to quantify the MPS distribution change in the periprosthetic femora, the average values of the MPS were computed for each ROI (Fig. 5). A decrease in the average MPS value after THA was proximally proved for both periprosthetic femora, pointing at stress shielding in this area.
In the diaphyseal and the distal ROI, the SPIRON does not cause any change in the MPS distribution at all. The BICONTACT, on the other hand, causes an additional slight decrease in the average MPS value in the diaphyseal ROI, but distally there is no change either.

Thus, it has been numerically proved that the stress shielding areas in the periprosthetic femur heavily depend on the prosthesis type used.

\section{DISCUSSION}

In general, stress shielding is a current problem in implant technology and specifically in artificial hip joints. It is one of the factors causing aseptic loosening of certain prostheses. Before, stress shielding was only suspected by biomechanical considerations (different stiffness of prostheses and bone) and clinical observations (bone resorption in the periprosthetic areas). Now, however, NFEA has been established as a suitable computing method to show stress shielding by examining the decrease in the load distribution after THA. 


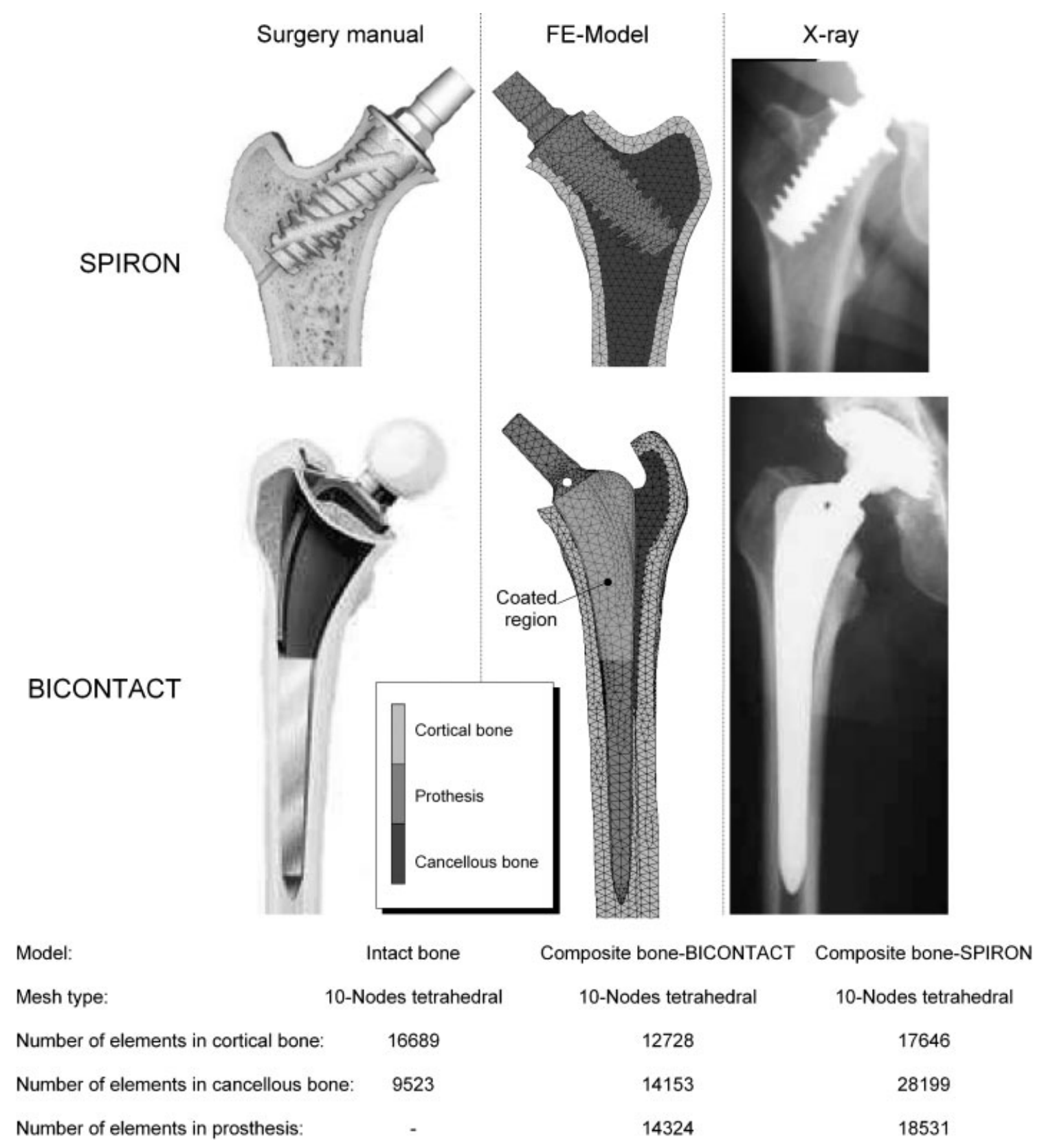

Fig. 2 Finite element modelling of the investigated composite bone prosthesis

Prendergast and Taylor [10], for example, compared the stress distribution in the proximal-medial femur after implantation of prostheses with different elastic moduli, while Mattheck et al. [11] analysed the impact of a hollow prosthesis on the stress distribution in the proximal femur and detected a reduction in stress shielding. Moreover, Taylor et al. [12] computed the periprosthetic stresses of different surface modifications of a femoral prosthesis component. They demonstrated higher stresses surrounding a 'press-fit' prosthesis in comparison with cemented or hydroxylapatite-coated stems.
To the authors' knowledge, the study by Tai $e t$ al. [13] is the only numerical investigation comparing an uncemented long-stem prosthesis with a femoral neck prosthesis regarding the computed stress distribution in the periprosthetic bone. They reported that stress shielding was eliminated using the cervio-trochanteric femoral component instead of a conventional anatomic stem.

However, Tai et al. [13], and other researchers as well $[\mathbf{1 8 - 2 1}]$, examined only half the femur in their numerical investigations. According to Duda et al. $[\mathbf{1 6}, \mathbf{2 2}]$, this does not correspond to 


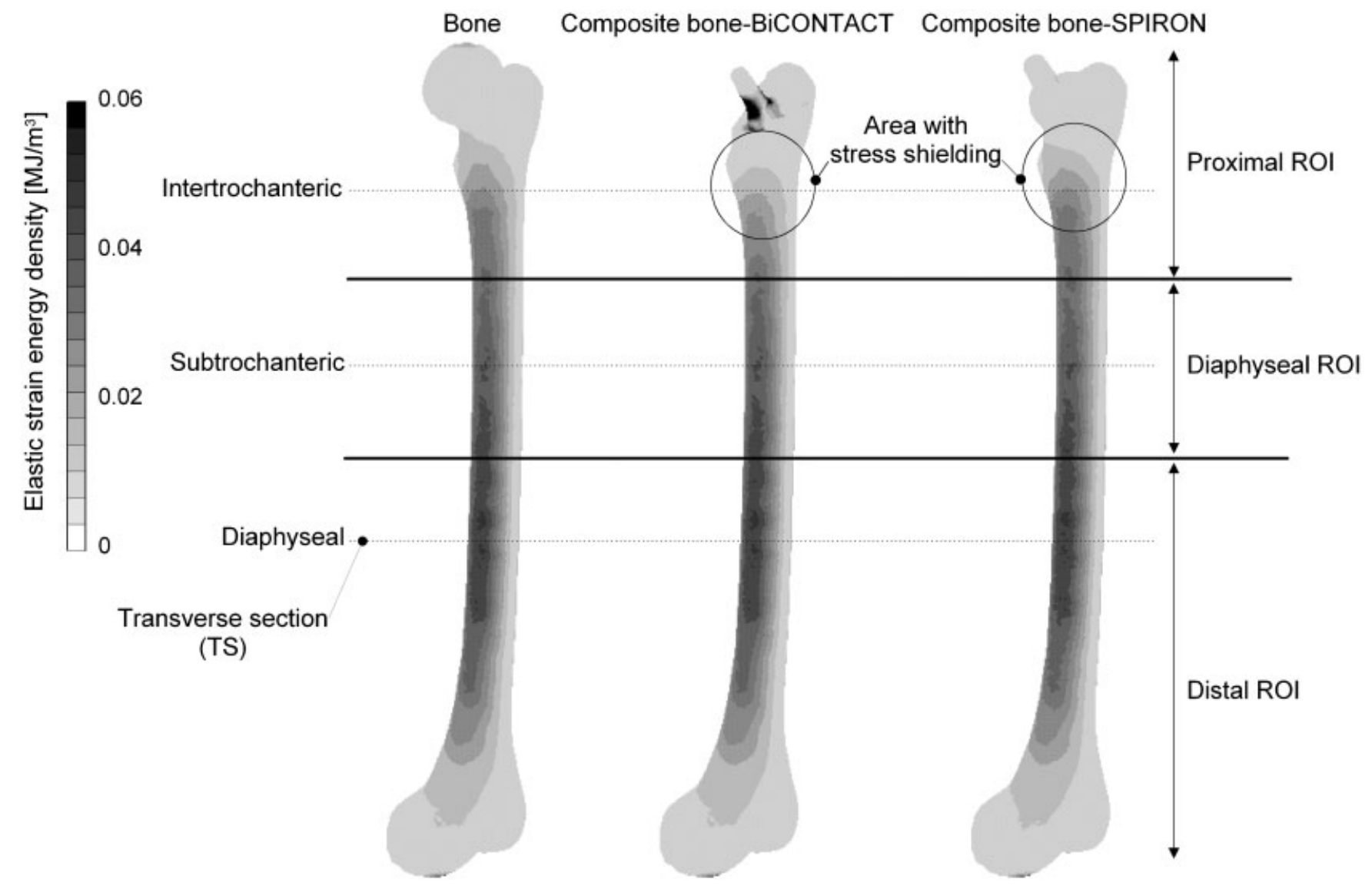

Fig. 3 Strain energy density in the intact femur (left), with a conventional uncemented prosthesis (middle), and with a femoral neck prosthesis (right)

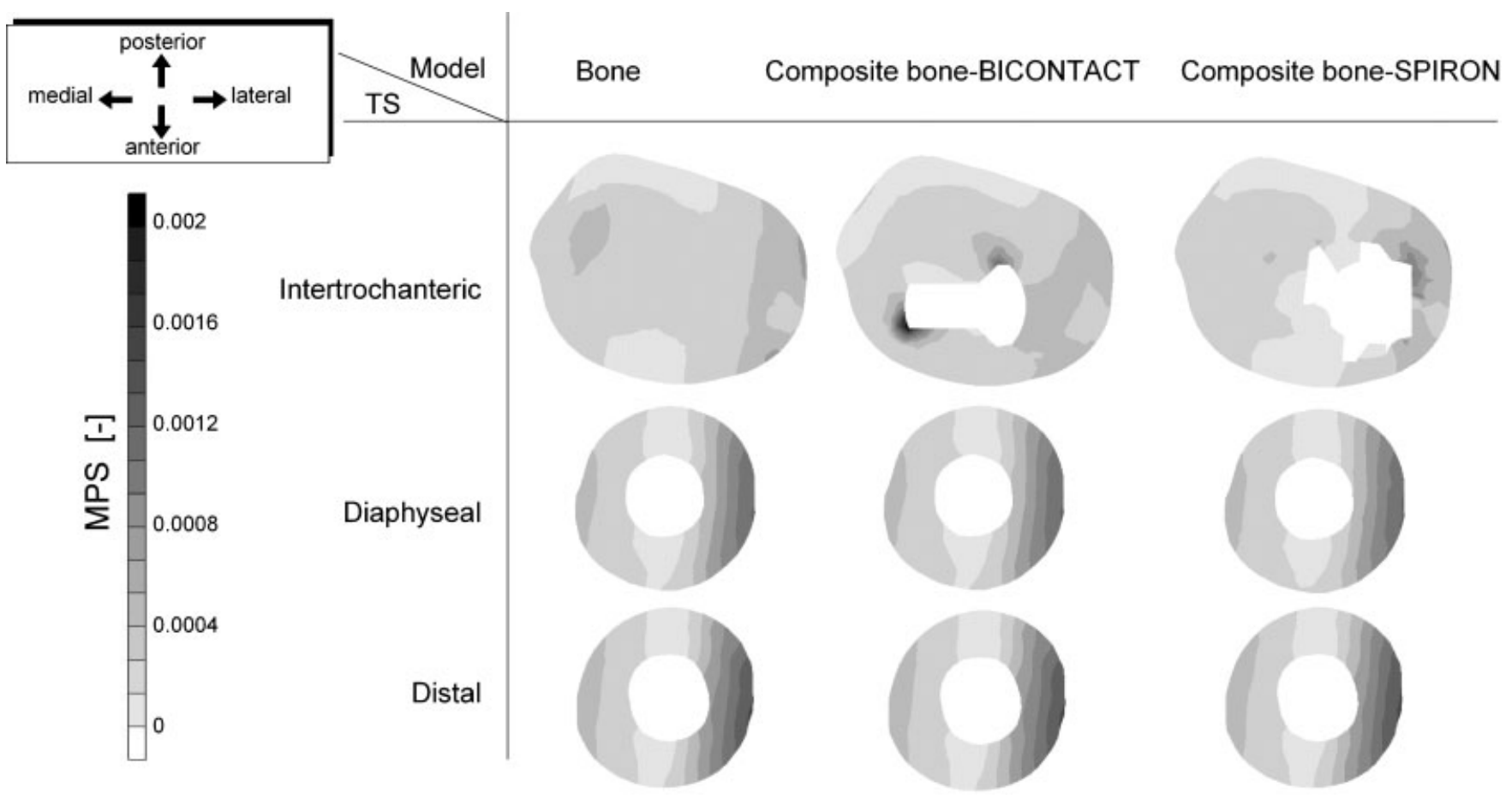

Fig. 4 Distributions of MPS in the TS across the bones 


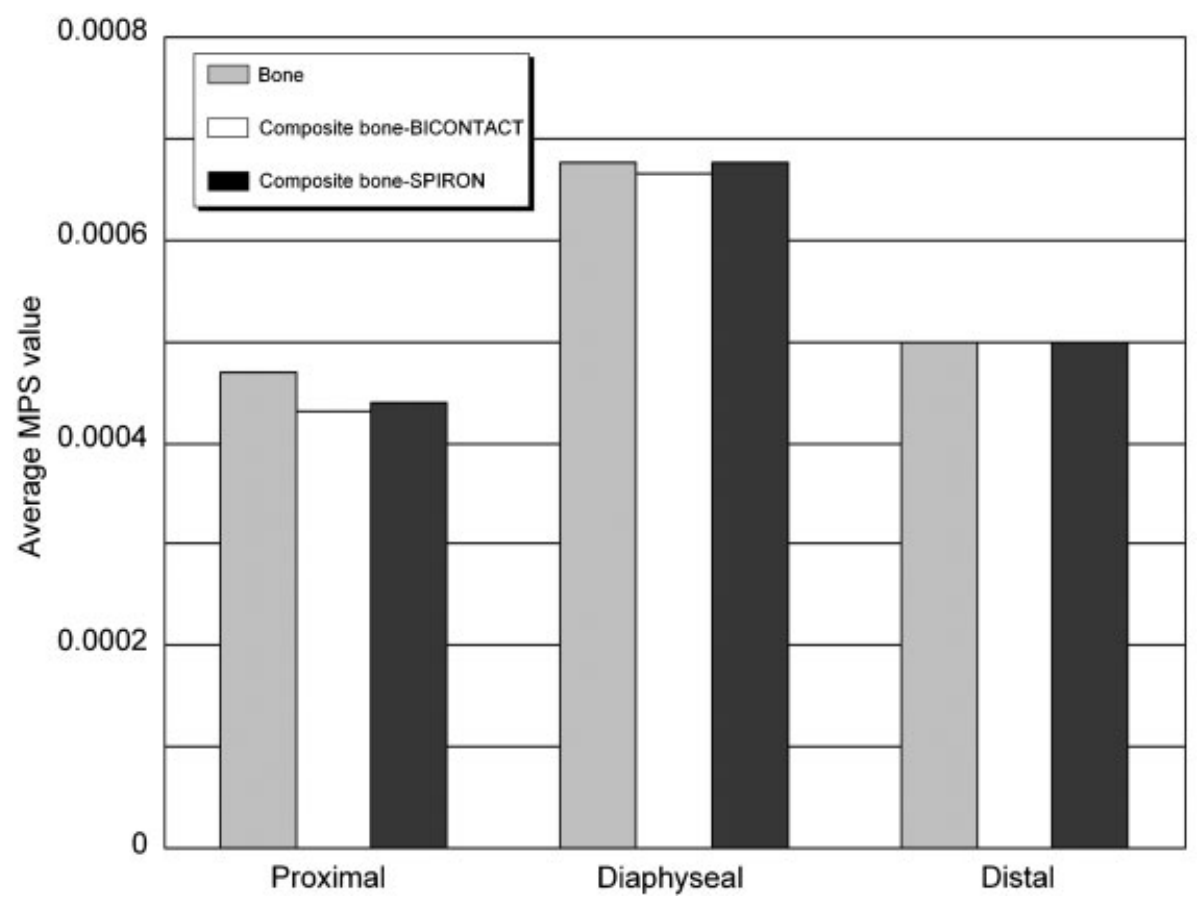

Fig. 5 Calculated average MPS values in the ROI

the physiological facts. Thus, the whole femur has been studied in the present study, as has been done in other works $[16,23,24]$. In this way, definite statements about the load situation in the distal part can be made.

Apart from that, Tai and co-workers [13] simplified the geometry of the prosthesis investigated. For example, they modelled the screws of the cerviotrochanteric component as pins. Such simplifications were made in the study by Taylor et al. [24] as well, wherein the authors numerically computed the bone remodelling stimulus in the periprosthetic bone that was provided with the thrust plate prosthesis. This stimulus represents the difference between the load situations in the intact and the periprosthetic femora. In the present study, an exact model of the SPIRON was made, and, by detailed examination of the screw thread, it was demonstrated that in the bone surrounding the thread there is a decrease in the load, implying stress shielding (Fig. 4). Thus, the numerical results prove that the use of the real geometry, and here especially the thread shape, is crucial for the computation of the load distribution in the bone surrounding the prosthesis.

Furthermore, the application of muscle forces is highly relevant for a correct computation of the load distribution in the bone $[\mathbf{1 6}, \mathbf{2 3}]$. In several studies it was assumed that the muscle forces may be pooled into one force with the greater trochanter as the acting point $[\mathbf{1 3}, \mathbf{1 8 - 2 0}]$. In the present study, calculations were made with the actual muscle forces because by this means the physiological situation can be depicted accurately. However, only a reduced muscle system was used, obtained from Heller et al. [17], to save time in the modelling process. The same reduced system was also used by Goetzen et al. [21], while Taylor et al. [24] took all muscle forces into account.

Many researches $[\mathbf{1 3}, 16,18-21]$ have not considered the anisotropy of the femoral bone, although numerous experiments have proven that the femur has no isotropic elasticity properties $[\mathbf{1 4}, \mathbf{2 5}, \mathbf{2 6}]$. In order to have a realistic simulation of the load distribution in the femur, a description of the bone elasticity properties as exact as possible is crucial. Hence, orthotropic properties were assigned to the cortical bone within the present study. The cortical bone is considerably stiffer than the cancellous bone, and thus it carries more load. On account of this, the authors simplified the depiction of the cancellous bone's elasticity properties, as others have done $[13,16,27]$, by using an isotropic material law.

These numerical investigations show that the uncemented long-stem prosthesis BICONTACT causes more changes in the load distribution in the proximal than in the diaphyseal area, compared with the physiological situation. This was proven in the clinical observations by Fritz et al. [28]. Apart from 
that, the present study shows that, owing to the implantation of a femoral neck prosthesis, stress shielding occurs in the proximal area only. This result was affirmed by numerical calculations $[\mathbf{1 3}$, 24] as well as clinical observations [29].

In summary, finite element analysis has been established as a reliable in silico method for demonstrating and computing stress shielding in prosthetic bones. However, accompanying clinical examinations are necessary to validate the computations and models used and to improve the material laws [30].

\section{ACKNOWLEDGEMENTS}

The study was realized in the framework of the collaborative research centre 599 'Sustainable degradable and permanent implants of metallic and ceramic materials'. The authors thank the German Research Foundation (DFG) for financial support of the project. The authors also wish to thank the companies Arge Medizintechnik GmbH and Aesculap $A G$ and Co. KG for providing CAD data of the SPIRON and BICONTACT femoral components.

\section{REFERENCES}

1 Adam, F. and Kohn, D. Computergestützte Entwicklung eines anatomischen Hüftprothesenschaftes. Magazin Forsch., 2000, 1, 41-48.

2 Sumner, D. R. and Galante, J. O. Determinants of stress shielding. Clinical Orthop. and Related Res., 1992, 274, 203-212.

3 Wolff, J. Das Gesetz der Transformation der Knochen, 1892 (Hirschwald, Berlin).

4 Arabmotlagh, M., Hennigs, T., and Rittmeister, M. Femoral periprosthetic bone remodelling to the proximal femur after implantation of custom made anatomic and standard straight stem hip prostheses. Z. Orthop. Grenzgeb., 2003, 141, 519-525.

5 Bergh, M. S., Muir, P., Markel, M. D., and Manley, P. A. Femoral bone adaptation to stable long-term cemented total hip arthroplasty in dogs. Vet. Surg., 2004, 33, 214-220.

6 Silva, M. J., Reed, K. L., Robertson, D. D., Bragdon, C., Harris, W. H., and Maloney, W. J. Reduced bone stress as predicted by composite beam theory correlates with cortical bone loss following cemented total hip arthroplasty. J. Orthop. Res., 1999, 17, 525-531.

7 Turner, A. W., Gillies, R. M., Sekel, R., Morris, P., Bruce, W., and Walsh, W. R. Computational bone remodelling simulations and comparisons with DEXA results. J. Orthop. Res., 2005, 23, 705-712.

8 Gervers, M., Nolte, I., Alt, F., Hauschild, G., MeyerLindenberg, A., and Fehr, M. Complications after implantation of a modular total hip prostheses compared with fixed head prostheses. Berl. Münchener Tierarztl. Wochenschr., 2002, 115, 412-419.

9 Senapati, S. K. and Pal, S. Finite element analysis: an effective tool for prostheses design. Trends Biomater. Artif. Organ, 2004, 17, 141-148.

10 Prendergast, P. J. and Taylor, D. Stress analysis of the proximo-medial femur after total hip replacement. J. Biomed. Engng, 1990, 12, 379-382.

11 Mattheck, C., Vorberg, U., and Kranz, C. Effects of hollow shaft endoprosthesis on stress distribution in cortical bone. Biomed. Tech. (Berl.), 1990, 35, 316-319.

12 Taylor, M., Tanner, K. E., Freeman, M. A., and Yettram, A. L. Cancellous bone stresses surrounding the femoral component of a hip prosthesis: an elastic-plastic finite element analysis. Med. Engng and Phys., 1995, 17, 544-550.

13 Tai, C. L., Shih, C. H., Chen, W. P., Lee, S. S., Liu, Y. L., and Hsieh, P. H. Finite element analysis of the cervico-trochanteric stemless femoral prosthesis. Clin. Biomech. (Bristol, Avon), 2003, 18, S53-S58.

14 Yoon, H. S. and Katz, J. L. Ultrasonic wave propagation in human cortical bone - II. Measurements of elastic properties and microhardness. J. Biomech., 1976, 9, 459-464.

15 Bini, F., Marinozzi, A., Marinozzi, F., and Patanè, F. Microtensile measurements of single trabeculae stiffness in human femur. J. Biomech., 2002, 35, 1515-1519.

16 Duda, G. N., Heller, M., Albinger, J., Schulz, O., Schneider, E., and Claes, L. Influence of muscle forces on femoral strain distribution. J. Biomech., 1998, 31, 841-846.

17 Heller, M. O., Bergmann, G., Kassi, J. P., Claes, L., Haas, N. P., and Duda, G. N. Determination of muscle loading at the hip joint for use in preclinical testing. J. Biomech., 2005, 38, 1155-1163.

18 Huiskes, R., Weinans, H., and Van, R. B. The relationship between stress shielding and bone resorption around total hip stems and the effects of flexible materials. Clin. Orthop., 1992, 124-134.

19 Fernandes, P. R., Folgado, J., Jacobs, C., and Pellegrini, V. A contact model with ingrowth control for bone remodelling around cementless stems. J. Biomech., 2002, 35, 167-176.

20 Weinans, H., Huiskes, R., and Grootenboer, H. J. Effects of material properties of femoral hip components on bone remodeling. J. Orthop. Res., 1992, 10, 845-853.

21 Goetzen, N., Lampe, F., Nassut, R., and Morlock, M. M. Load-shift-numerical evaluation of a new design philosophy for uncemented hip prostheses. J. Biomech., 2005, 38, 595-604.

22 Viceconti, M., Ansaloni, M., Baleani, M., and Toni, A. The muscle standardized femur: a step forward in the replication of numerical studies in biomechanics. Proc. Instn Mech. Engrs, Part H: J. Engineering in Medicine, 2003, 217, 105-110. 
23 Polgár, K., Gill, H. S., Viceconti, M., Murray, D. W., and O'Connor, J. J. Strain distribution within the human femur due to physiological and simplified loading: finite element analysis using the muscle standardized femur model. Proc. Instn Mech. Engrs, Part H: J. Engineering in Medicine, 2003, 217, 173-189.

24 Taylor, W. R., Ploeg, H., Hertig, D., Warner, M. D., and Clift, S. E. Bone remodelling of a proximal femur with the thrust plate prosthesis: an in vitro case. Computer Meth. in Biomechanics and Biomed. Engng, 2004, 3, 131-137.

25 Wirtz, D. C., Pandorf, T., Portheine, F., Radermacher, K., Schiffers, N., and Prescher, A. Concept and development of an orthotropic FE model of the proximal femur. J. Biomech., 2003, 36, 289-293.

26 Taylor, W. R., Roland, E., Ploeg, H., Hertig, D., Klabunde, R., and Warner, M. D. Determination of orthotropic bone elastic constants using FEA and modal analysis. J. Biomech., 2002, 35, 767-773.

27 Kabel, J., Van, R. B., Dalstra, M., Odgaard, A., and Huiskes, R. The role of an effective isotropic tissue modulus in the elastic properties of cancellous bone. J. Biomech., 1999, 32, 673-680.

28 Fritz, S. T., Volkmann, R., Winter, E., and Bretschneider, C. The BICONTACT hip endoprosthesis - a universal system for hip arthroplasty for traumatic and idiopathic indications: results after 10 years. Eur. J. Trauma - E - Supplement, 2001, 1, S18-S22.

29 Tai, C. L., Lee, M. S., Chen, W. P., Hsieh, P. H., and Liu, Y. L. Biomechanical comparison of newly designed stemless prosthesis and conventional hip prosthesis - an experimental study. Bio-Med. Mater. Engng, 2003, 15, 239-249.

30 Lengsfeld, M., Burchard, R., Gunther, D., Pressel, T., Schmitt, J., and Leppek, R. Femoral strain changes after total hip arthroplasty - patientspecific finite element analyses 12 years after operation. Med. Engng and Phys., 2005, 27, 649-654.

\section{APPENDIX}

\section{Notation}

$\mathrm{CAD} \quad$ computer aided design

CT

E

FEA

MPS

SED

THA computed tomography

Young's modulus

finite element analysis

maximal principal strain

strain energy density

total hip arthroplasty 